\title{
A note from the Editor-in-Chief
}

Nava Segev

Editor-in-Chief; Cellular Logistics; and Department of Biological Sciences; University of Illinois at Chicago; Chicago, IL USA

This is the fourth and last issue for the first year of our new journal, Cellular Logistics. In this issue we have a research paper, four addenda and a collection of three reviews together with an editorial highlighting a productive merge of two field fronts, micro and cell biology.

During this year, our budding journal was accepted for inclusion in PubMed Central. With the current issue, we can apply for inclusion in Medline. Inclusion in Medline would decrease the time between publication and appearance of papers in PubMed, and hopefully increase the number of submissions to the journal.

All this could not have been achieved without the help of our associate and acquisition editors, our editorial board, the support system of Landes Bioscience and the vote of confidence of all the contributors who submitted their work for publication.

Our goals for next year are to increase the scope of the journal and continue to provide a stage for reasoned debates on scientific disputes.

Nava Segev

Editor-in-Chief

Cellular Logistics 